East African Medical Journal Vol. 87 No. 5 May 2010

CEREBRAL VENOUS SINUS THROMBOSIS: A REPORT OF TWO CASES

C. K. Onyambu, MBChB, MMed (Diag. Radiol), Lecturer, Department of Diagnostic Imaging and Radiation Medicine, I. M. Muriithi, MBChB, MMed (Diag. Radiol), Tutorial Fellow, Department of Diagnostic Imaging and Radiation Medicine, and S. M. Ngare, MBChB, Postgraduate Resident, Department of Clinical Medicine and Therapeutics, College of Health Sciences, University of Nairobi, P.O. Box 19676-00202, Nairobi, Kenya

Request for reprints to: Dr. C. K. Onyambu, P.O. Box 88-00202, Nairobi, Kenya

\title{
CEREBRAL VENOUS SINUS THROMBOSIS: A REPORT OF TWO CASES
}

\author{
C. K. ONYAMBU, I. M. MURIITHI and S. M. NGARE
}

SUMMARY

\begin{abstract}
Cerebral venous sinus thrombosis is an uncommon yet potentially life threatening condition which is often under-diagnosed or misdiagnosed because of the variability of the clinical signs and symptoms. The imaging findings are often subtle thus the need for a high index of suspicion. The superior sagittal and transverse sinuses are the most frequently involved. The correct diagnosis of cerebral venous thrombosis relies on neuroimaging studies. The two cases reported highlight the fact that the radiologist may be the first clinician to suspect and diagnose this condition. This ultimately affects prognosis as early diagnosis and treatment reduces morbidity and mortality.
\end{abstract}

\section{INTRODUCTION}

Cerebral venous sinus thrombosis is a challenging condition because it is uncommon and often associated with non-specific symptoms and signs. It is often unrecognised at initial presentation and the imaging features can be subtle and variable. It has been more frequently diagnosed in the past $10-15$ years partly due to greater awareness among physicians and also due to improved non-invasive imaging techniques (1). The radiologist plays a crucial role in patient care by providing early diagnosis through correct interpretation of imaging studies.

We report on two patients who presented with varied neurological signs but in whom sinus thrombosis had not been suspected clinically. Sinus thrombosis was subsequently revealed on neuroimaging. Treatment wasinstituted with clinical improvement. Follow up was done.

The purpose of this article is to raise the index of suspicion for cerebral venous sinus thrombosis given the variability of clinical signs.

\section{CASE REPORTS}

Case 1: A34-year-old housewife presented with a one day history of generalised tonic clonic seizures. The seizures were noted to last 5 minutes and followed by post-ictal confusion for 10 minutes. She had experienced four seizures prior to admission. She also gave history of headache for two months. The headache was global in nature with no relieving or aggravating factors. There was no history of vomiting or visual disturbances. She gave no history of fever or trauma. She was a para $4+0$ and had been on oral contraceptives for three years.

On admission, she had a Glasgow Coma Scale of $3 / 15$, was in post-ictal state, and upon improvement of her neurological status, was later noted to have left upper limb weakness (UMN grade 3). Her blood pressure was $110 / 70 \mathrm{mmHg}$, pulse rate was 77 beats per minute and temperature $36.4^{\circ} \mathrm{C}$. Coagulation screen showed a PTT of 19 sec, PTI of 79\%, INR of 1.32 and APTT of 45 seconds. Haemogram was normal.

Head CTscan donerevealed bilateral parasagittal infarcts in the pre-contrast images, the right showing haemorrhagic transformation (Figure 1A). Hyperdensity of the superior sagittal sinus, effacement of the sulci and gyri and attenuation of the lateral and third ventricles was seen. Contrast enhanced CT scans showed the empty delta sign in the superior sagittal sinus (Figure 1A and B.). This was better demonstrated on sagittal (Figure 1C) reformatted scans which showed lack of enhancement in the superior sagittal sinus. A diagnosis of superior sagittal sinus thrombosis with bilateral parasagittal infarcts, the right being haemorrhagic was made.

The patient was managed with I.V heparin and epanutin. She did well on treatment and was discharged on oral warfarin and tegretol through the medical outpatient clinic. She was advised to continue with warfarin for three months. 


\section{Figure 1}

Axial contrast enhanced CT $(A)$ shows the empty delta sign (open straight arrow) in keeping with thrombosis of the superior sagittal sinus. Bilateral parasagittal infarcts are seen with haemorrhagic transformation on the right (solid arrow). Axial (B) and sagittal reformatted $(C)$ contrast enhanced $C T$ images confirm lack of enhancement in the superior sagittal sinus (open curved arrows)

\section{Case 1 - CT scan images}

A

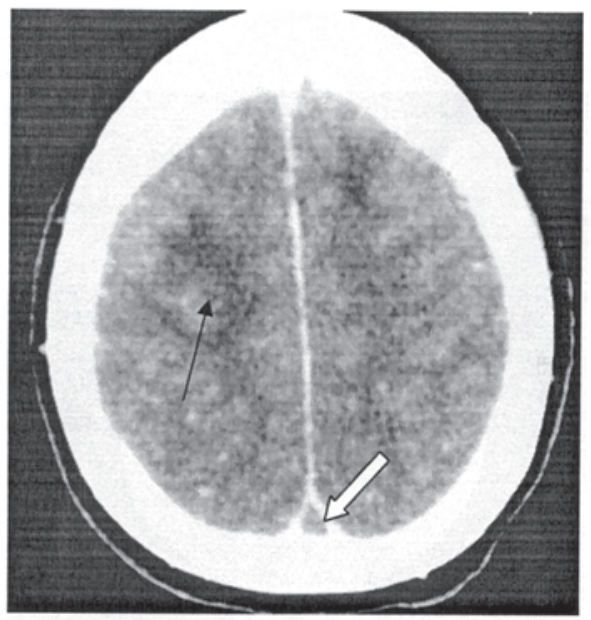

B

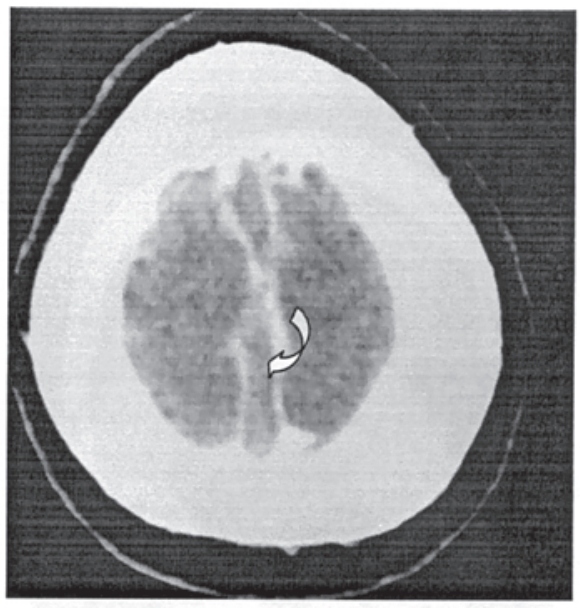

C

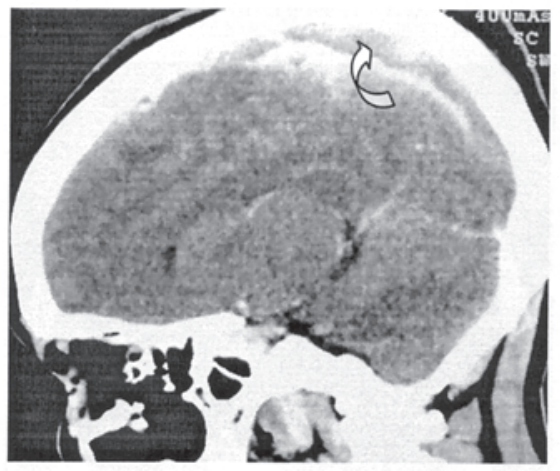

Figure 2

Non-enhanced axial CT scan image (A) showing a serpiginous hyperdensity (solid arrow) on the left parasagittal cerebral convexity draining towards the superior sinus ('dense cord'). Contrast enhanced coronal (B) and right parasagittal (C) CT scan images showing the 'empty delta sign' in the superior sagittal and right transverse sinuses (curved open arrows)

Case 2 - CT scan images

A

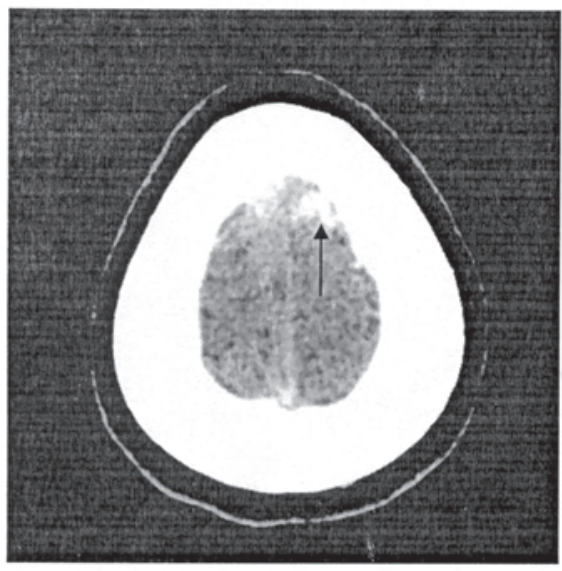

B

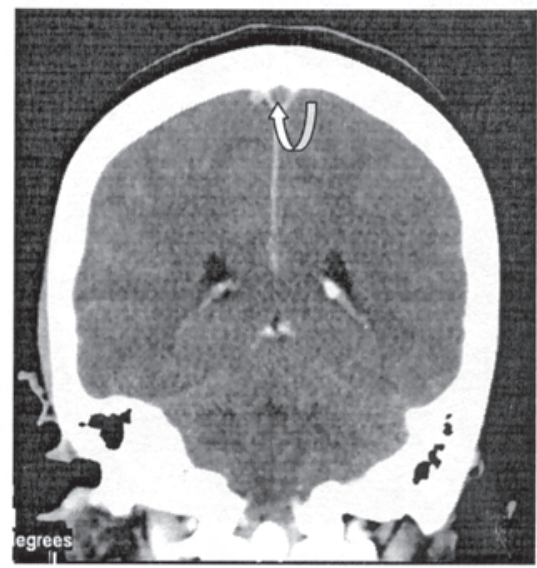

C

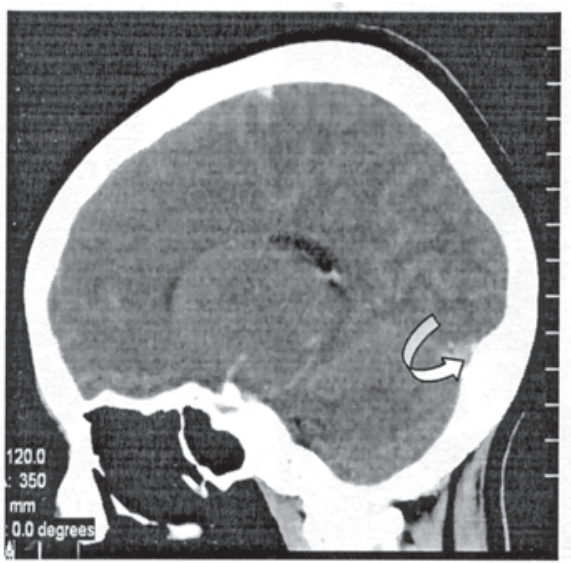


Case 2: A33-year-old female patient was admitted with a two week history of severe headaches. There was no history of photophobia, neck pains or convulsions. She was a para $2+0$ and gave no history of oral contraceptives use or recent preceding pregnancy.

On examination, she had a Glasgow Coma Scale of $15 / 15$, with neck stiffness and papilloedema on fundoscopy. Her blood pressure was $100 / 70 \mathrm{mmHg}$ and pulse rate was 96 beats per minute.

Coagulation screen done showed a PTT of 19 sec, PTI of $75 \%$, and INR of 1.39. Platelet count was $311 \times 10^{9}, \mathrm{WBC}$ count was $9.74 \times 10^{9}$ and $\mathrm{Hb}$ was $17.5 \mathrm{~g} / \mathrm{dl}$. Serology for HIV, hepatitis B surface antigen, hepatitis C antibody, rheumatoid factor, VDRL, ANA were negative. Lupus anticoagulant was positive.

HeadCT scan done showed effacement of cerebral sulci bilaterally and hyperdensity within the superior sagittal and right transverse sinuses on the nonenhanced scans ('dense clot sign'). Also noted on the non-enhanced scans was a serpiginous hyperdensity on the left parasagittal cerebral convexity draining into the superior sagittal sinus ('dense cord sign') (Figure 2A). Contrastenhanced scans revealed theempty delta signin the superior sagittal and right transversesinuses (Figures 2B and C). Adiagnosis of superior sagittal and right transverse sinus thrombosis was then made. An MRI and 2D time-of-flight MR venogram were done which confirmed absence of flow void (Figure 3C) and absence of flow (Figure 3D) within the superior sagittal and right transverse sinuses.

The patient was managed with I.V heparin followed by oral warfarin. She was discharged on warfarin for three months to be followed up in the medical outpatient clinic.

Figure 3

Mid sagittal (A) and axial (B) non contrast enhanced T1 weighted images showing a filling defect (solid arrows) in the superior sagittal sinus. Coronal T2 weighted image (C) showing absence of flow void in the superior sagittal sinus (straight open arrow) and right transverse sinus (curved open arrow). 2D time-of flight MR venogram coronal view (D). Showing nonvisualization of the right transverse (and proximal right sigmoid) and superior sagittal sinuses

\section{Case 2 - MR images}

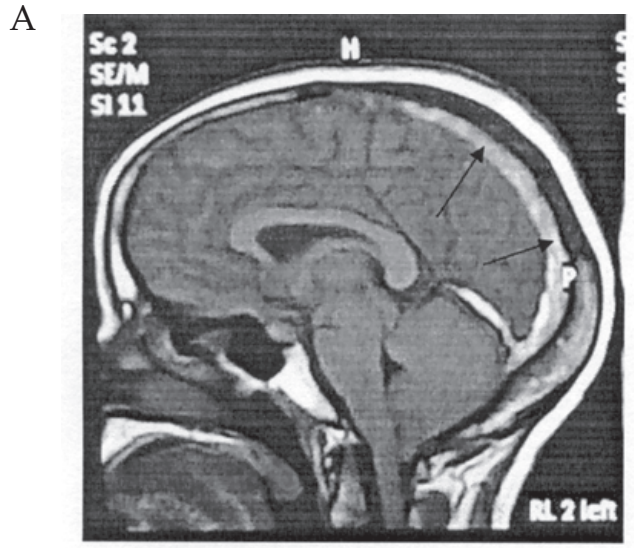

B
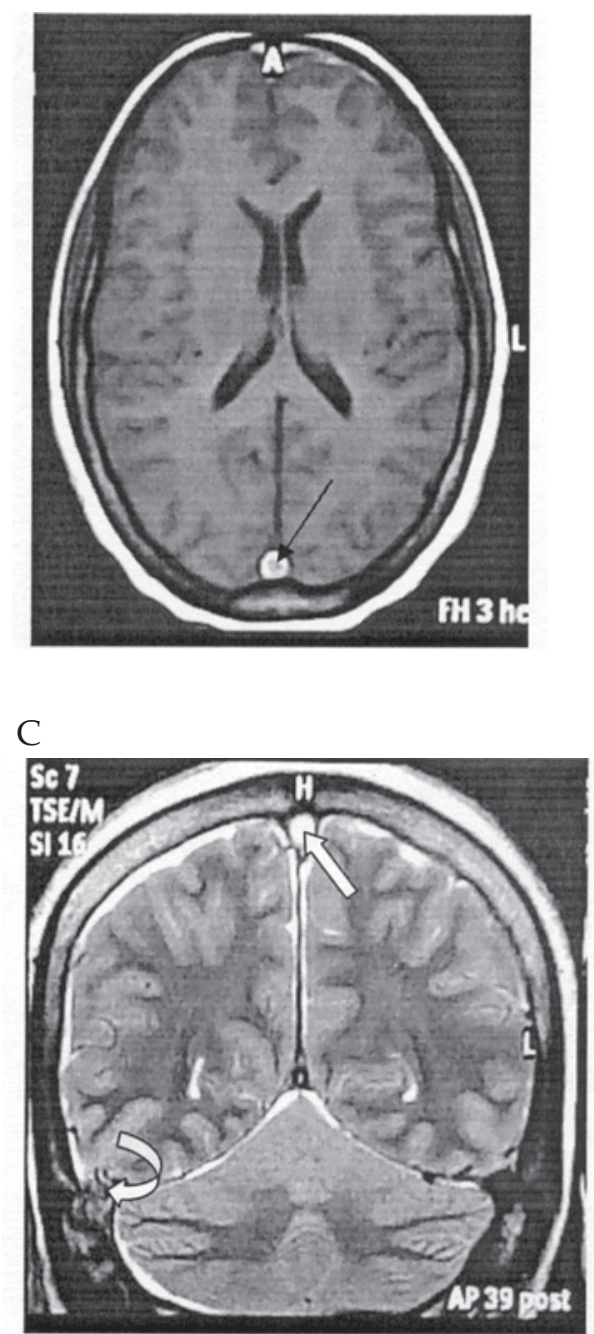

$\mathrm{D}$

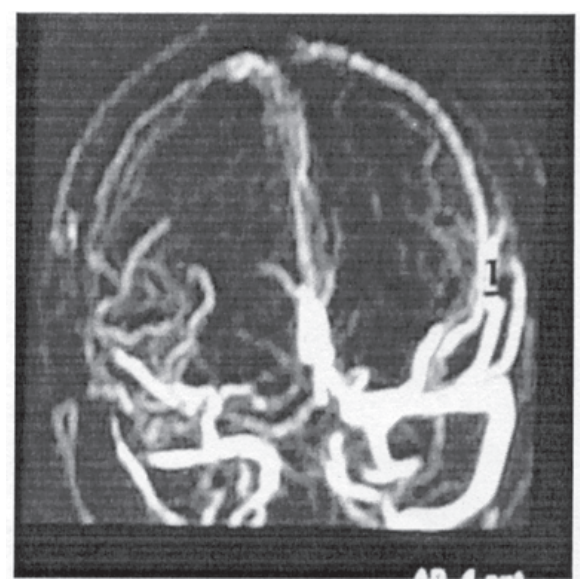

DISCUSSION

Cerebral venous sinus thrombosis is commonly under-diagnosed because of the variable clinical presentation. It may affect any age although it is more common in women in the age group of 20 to 35 years due to pregnancy, puerperium and oral contraceptive use (1). 
Theaetiology of cerebral venoussinus thrombosis includes local causes such as head injury, craniotomy, meningitis and local sepsis such as mastoiditis. Systemic causes include dehydration, septicaemia, pregnancy and puerperium, inflammatory bowel disease, SLE and malignancy. Blood dyscracias like leukemia, thrombocythemia and sickle cell disease are also causes of thrombosis. Drugs associated with thrombosis include the oral contraceptive pill, hormone replacement therapy and ecstacy. Coagulopathies like protein S, C and antithrombin III deficiencies also cause cerebral venous sinus thrombosis. In 20-35\% of cases, no aetiologic factor can be identified (1). In the first case that we reported the thrombosis was possibly related to oral contraceptive use. In the second case the thrombosis was possibly related to the presence of the lupus anticoagulant.

Clinical symptoms and signs arenon-specific and includeheadache, focal neurological deficits, seizures, isolated benign intracranial hypertension-like picture, impaired level of consciousness and papilloedema.

Cerebral venous sinus thrombosis commonly involves the superior sagittal sinus followed by transverse sinuses (1). In a third of cases, more than one sinus is involved. The sinuses involved in our cases were superior sagittal and transverse sinuses.

Non-enhanced CT scans remain the technique of choice for screening patients with non-specific clinical presentation and a low suspicion of dural sinus thrombosis (2). Non-enhanced CT scans may show direct and indirect signs of cerebral venous sinus thrombosis. Direct signs are seen in only a third of cases (8) and include thrombus in the dural sinus giving the 'dense clot sign'. There could also be the 'dense cord sign' which represents direct visualization of a thrombosed cortical vein that is seen as linear hyperdensity. Indirect signs include diffuse brain oedema, decreased ventricular size and venous infarction. Venous infarction is seen as infarction not conforming to a major arterial vascular territory, multipleisolated lesions and involvement of the subcortical region with sparing of the cortex. The infarct may behaemorrhagic or non-haemorrhagic (3). Haemorrhagic infarcts affect the cortex and adjacent white matter and occur in about $10-50 \%$ of cases (6). Our first reported case showed parasagittal infarcts with haemorrhagic transformation on the right.

Contrast enhanced CT scans show a filling defect (thrombus) in the dural sinus with peripheral dural enhancement ('empty delta sign'), which may be seen five days to two months from onset of symptoms. This sign is only demonstrated in $10-20 \%$ of cases (1). In 10-30\% of cases, the findings on either non-enhanced or contrast enhanced CT scans are negative (9). Therefore, in clinically suspected cases,
CT venography or MRI with MR venogram should be done. CT venography shows the thrombus as a filling defect (4).

MRI and MR venography have been the noninvasive imaging technique of choice $(3,5)$. They are often used as the initial diagnostic test for clinically suspected cases. Conventional MRI shows the thrombus as absence of a flow void, which is best seen on FLAIR and T2-weighted spin-echo images. Venous infarcts, which may be haemorrhagic or nonhaemorrhagic, may be visualized. Contrast enhanced T1-weighted MRI may show the empty delta sign due to non-enhancing thrombus surrounded by enhancing dura.

MR venography using the time-of-flight (TOF) or phase contrast techniques are often diagnostic. They show the thrombus as an area of lack of flow but are subject to flow-related imaging artifacts because these techniques use MR flow phenomena to generate contrast. Contrast enhanced MR venography, which utilises luminal filling by contrast material and is thus less likely to be affected by complex flow, has been shown to provide better diagnostic information than 2D TOF-MR venography (5).

CT venography has been shown to be superior to 2D time-of-flight or phase contrast MR venography techniques (2). Direct comparison studies between CT venography and contrast enhanced MR venography are not yet available (8).

Analysis of cerebrospinal fluid (CSF), in the appropriate clinical context, is important to exclude meningitis and subarachnoid haemorrhage as they may have similar symptoms.

Anticoagulation is the treatment of choice in patients with cerebral venous sinus thrombosis. Initial treatment with IV heparin is the first line of treatment (6).

Intravenous heparin has been shown to be beneficial evenin patients whohave had haemorrhagic infarction and is only withheld in patients with large haemorrhagic venous infarcts with significant mass effect (10). This is then followed by oral warfarin. Local thrombolysis should be performed in patients who show deterioration despite adequate heparinisation (7). Oral anticoagulants should be continued for at least three to six months.

The purpose of this article is to raise the index of suspicion for cerebral venous sinus thrombosis given the variability of clinical signs. Correct diagnosis of dural sinus thrombosis can be made using neuroimaging tools. We hope that after reading this article, the readers will be able to diagnose cerebral venous sinus thrombosis more accurately using the optimum imaging tool. 


\section{REFERENCES}

1. Ameri, A. and Bousser, M.G. Cerebral venous sinus thrombosis. Neural. Clin. 1992; 10: 87-111.

2. Ozsvath, R.R., Casey, S.O., Lunstrin, E.S., et al. Cerebral venography: comparison of CT and MR projection venography. AJR. 1997; 169: 1699-1707.

3. Zimmerman, R.D. and Ernest, R.J. Neuroimaging of cerebral venous thrombosis. Neuro. Clin. North Am. 1992; 2: 463-485.

4. Casey, S.O., Alberico, R.A., Patel, M., et al. Cerebral CT venography. Radiology. 1996; 198: 163-170.

5. Lee, S-K. and terBrugge, K.G. Cerebral venous thrombosis in adults: the role of imaging evaluation and management. Neuro. Clin. North Am. 2003; 13: 139-152.

6. Provenzale, J.M., Joseph, G.J. and Barboriak, D.P. Dural sinus thrombosis: Findings on CT and MR imaging and diagnostic pitfalls. AJR. 1998; 170: 777783.
7. Vogi, T.J., Bergman, C., Villringer, A., et al. Dural sinus thrombosis: value of venous MRA for diagnosis and follow-up. AJR. 1994; 162: 1191-1198.

8. Poon, C. S., Chang, J. K., Swarnkar, A., Johnson, M.H. and Wasenko, J. Radiologic diagnosis of cerebral venous sinus thrombosis: Pictorial review. AJR. 2007; 189: S64-S75.

9. Virapongse, C., Cazenave, C., Quisling, R., Sarwar, M. and Hunter, $\mathrm{S}$. The empty delta sign: frequency and significance in 76 cases of dural sinus thrombosis. Radiology. 1987; 162: 779-785.

10. Mehta, S.R., Muthukrishnan, J., Vardarajulu, R. and Gupta, A. Cerebral venous sinus thrombosis: A great masquerader. MJAF1. 2004; 60: 299-301. 